\title{
NOTE ON A PAPER BY SINGH AND DHALIWAL
}

\author{
by W. E. WILLIAMS
}

(Received 21st May 1980)

In a recent paper (1) Singh and Dhaliwal present an analysis which purports to solve a pair of dual integral equations which occur in crack theory and the object of this note is to demonstrate that their analysis is erroneous.

In (1) the integral equation

$$
\int_{0}^{\infty} \xi \psi(\xi)\left(1-\xi^{2} \delta^{2} \operatorname{cosech}^{2} \xi \delta\right) \cos \xi x d \xi=f(x), \quad 0<x<a,
$$

is rewritten as

$$
\int_{0}^{\infty} \xi \psi(\xi) \frac{\partial}{\partial \delta}\left(\frac{-1}{\delta}+\xi \operatorname{coth} \xi \delta\right) \cos \xi x d \xi=\frac{f(x)}{\delta^{2}}, \quad 0<x<a
$$

and this equation is then integrated with respect to $\delta$ to give

$$
\int_{0}^{\infty} \xi \psi(\xi)\left(\frac{-1}{\delta}+\xi \operatorname{coth} \xi \delta\right) \cos \xi x d \xi=\frac{-f(x)}{\delta}+g(x) .
$$

The step from equation (2) to equation (3) is only valid if $\psi$ is independent of $\delta$ and examination of equation (1) suggests that this is most unlikely to be the case and the actual $\psi$ obtained in (1) is not independent of $\delta$. This error invalidates the remainder of the analysis of (1) and there seems to be no simple way of rectifying it so as to obtain an analytic solution of the integral equations considered.

\section{REFERENCE}

(1) B. M. Singh and R. S. Dhaliwal, Proc. Edinburgh Math. Soc. 22 (1979), 213-215.

Mathematics Department

UNIVERSITY OF SURREY 